Document downloaded from:

http://hdl.handle.net/10251/168796

This paper must be cited as:

Melgarejo, JC.; Cogollos, S.; Guglielmi, M.; Boria Esbert, VE. (2020). A New Family of Multiband Waveguide Filters Based on a Folded Topology. IEEE Transactions on Microwave Theory and Techniques. 68(7):2590-2600. https://doi.org/10.1109/TMTT.2020.2989111

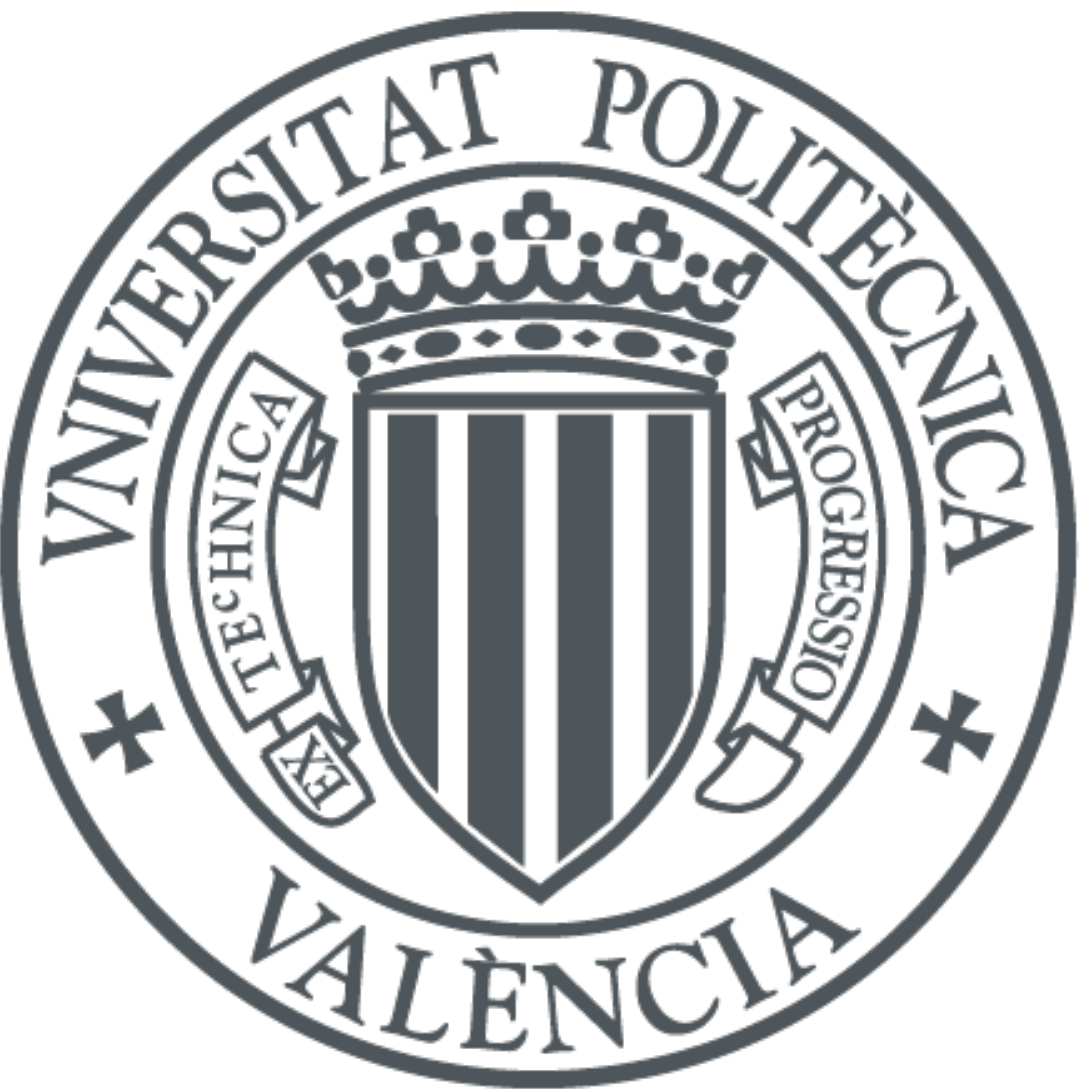

The final publication is available at

https://doi.org/10.1109/TMTT.2020.2989111

Copyright Institute of Electrical and Electronics Engineers

Additional Information 


\title{
A New Family of Multi-Band Waveguide Filters Based on a Folded Topology
}

\author{
Juan C. Melgarejo, Santiago Cogollos, Member, IEEE, Marco Guglielmi, Life Fellow, IEEE and Vicente E. Boria, \\ Fellow, IEEE,
}

\begin{abstract}
In this paper we describe a new family of multi-band waveguide filters based on a folded topology. The folded topology allows the use filters of different order, and the introduction of transmission zeros. The design of the multi-band filter is based on the Aggressive Space Mapping (ASM) technique and is able to take into account manufacturing details, like round corners and tuning elements. The filter structure is validated by designing, manufacturing and measuring a tri-band filter. The agreement between simulations and measurements is shown to be excellent, thereby fully validating both the filter topology, and the design process.
\end{abstract}

Index Terms-Circuit design, microwave filter, multi-band filter, space mapping, optimization, tri-band filter, waveguide filters.

\section{INTRODUCTION}

$\mathbf{M}$ ULTI-BAND filters are becoming interesting components for the implementation of advanced satellite payloads [1], [2]. Several channels, which are non-contiguous in frequency, may be amplified with one single amplifier and sent to a coverage area through one beam. Therefore, a multiband filter is required to reject interleaving channels from other beams. This greatly simplifies the system architecture [3].

Typically, there are four common approaches to design a multi-band filter [4]. The first approach is to use circulators to add/drop each channel. The advantage of this approach is the modularity and the simplicity of the design process. The disadvantages are cost, size and insertion loss (IL). Another solution is to cascade a wide band-pass and one (or more) band-stop filter(s). The advantages are the same as those of the first approach: simplicity and modularity. However, this approach requires a rather large footprint. The third solution is to use in-band transmission zeros to divide a higher-order bandpass filter in several lower-order channels [5], [6]. The fourth solution is to use resonators with multiple modes. This approach provides the most compact solution. However, it can only be used if the pass bands are sufficiently separated. There

Manuscript received Month DD, YYYY; revised Month DD, YYYY; accepted Month DD, YYYY.

This work was supported by funds from "La Caixa" Foundation (Code: B004442), and from Agencia Estatal de Investigación (AEI) and Unión Europea through Fondo Europeo de Desarrollo Regional - FEDER - "Una manera de hacer Europa" (AEI/FEDER, UE), under the Research Project TEC2016-75934-C4-1-R.

Juan C. Melgarejo, Marco Guglielmi, Santiago Cogollos and Vicente E. Boria are with Departamento de Comunicaciones, iTEAM, Universitat Politècnica de València, E-46022, Spain (e-mails: juamelle@teleco.upv.es,_marco.guglielmi@iteam.upv.es, sancobo@dcom.upv.es and vboria@dcom.upv.es ). are, indeed, a number of examples in the literature of dualmode and triple-mode resonators implementations of multiband filters. In [7] and [8], for instance, a triple-conductor combline resonator is used to implement dual-band filters. A dual-mode filter structure was also used to generate a dualband filter in [9]. In [10] a new class of dual-band filters and diplexers, based on dual-band resonators was also discussed. Finally, a novel configuration of dual-band filters using sidecoupled elliptical cavity resonators was reported in [11]. Although multi-mode resonator implementations are attractive, they all have one fundamental drawback, namely, the difficulty of introducing transmission zeros. The fact that transmission zeros can be easily implemented in standard dual-mode filters is indeed well known [11], but it has not been demonstrated so far for multiple-mode resonator implementations of multiband filters [4].

Recently, the well-known multiplexer design technique described in [12] was adapted to the design of a tri-band waveguide filter [13]. Fig. 1 shows the filter schematic diagram and Fig. 2 shows the actual body of a tri-band filter example. The manifold approach allows to introduce as many channels as required, without any limitations in terms of channel spacing. There are, however, some limitations regarding the physical realization of these filters, namely:

1) The physical length of every channel must be equal. In order to guarantee this requirement, stubs $\left(\theta_{1}, \ldots, \theta_{N}\right.$ of Fig. 1) must be added to the manifold, thus increasing the size of the filter.

2) The constraint in length is especially challenging when dealing with channels with different orders or when the channels have very different bandwidth specifications.

3) It is not possible to introduce cross-couplings between non-adjacent resonators in the same channel.

In this context, the objective of this paper is to significantly extend the results described in [13] by proposing a flexible and more general structure that does not have any of the three limitations just discussed and, at the same time, it maintains the advantages of the original method (i.e. flexibility in the number of channels, frequency spacing, etc.). This objective is achieved by proposing a new folded topology. This new topology will be tested through the design of a tri-band filter that has different specifications for each individual pass band.

The paper is organized as follows: Section II discusses the new folded topology. Section III summarizes the design procedure and section IV is focused on the specific design of the individual channels. In Section V, we present in detail 


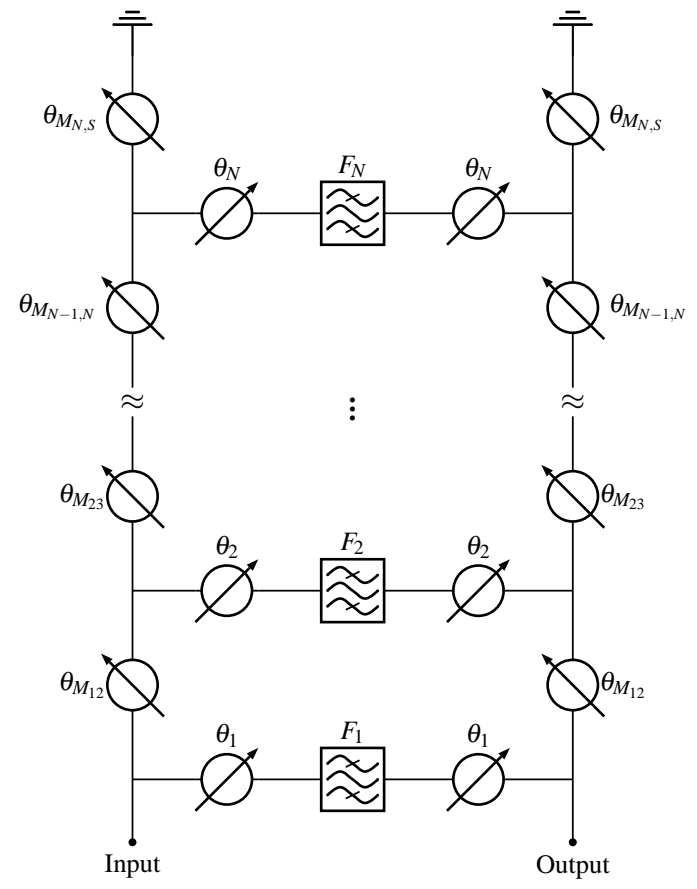

Fig. 1: Filter schematic of a multi-band filter using the manifold approach.

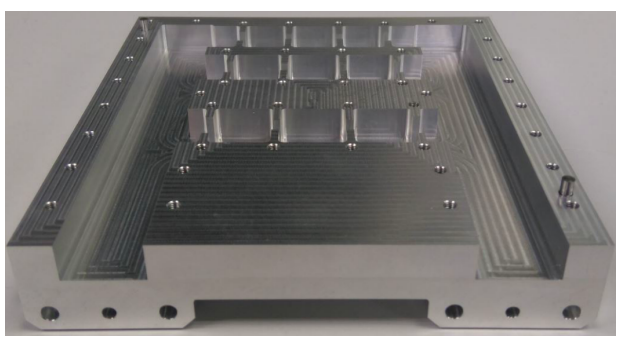

Fig. 2: Body of the manufactured tri-band filter proposed in [13] using the manifold approach.

the design procedure of the tri-band filter example. The same procedure is applicable also to any other set of multi-band filter specifications. In Section VI we discuss important issues of practical importance. In Section VII, a new method to increase the accuracy of the simulations is introduced. In Section VIII, we compare the simulation with measurement results. Finally, we summarize the results obtained in section IX.

\section{Folded TOPOLOGY}

The filters that we use for every channel of the multi-band filter are folded in the E-plane as shown in Fig. 3. The main advantages of the new folded topology are as follows:

1) It removes the constraint of the previous design concerning the length of the filters. Since the filters are folded in the center, the length and the order of the individual filters can be completely different. Using the folded topology that we propose, each channel can have very different specifications (in terms of order or bandwidth) without increasing the complexity of the design.
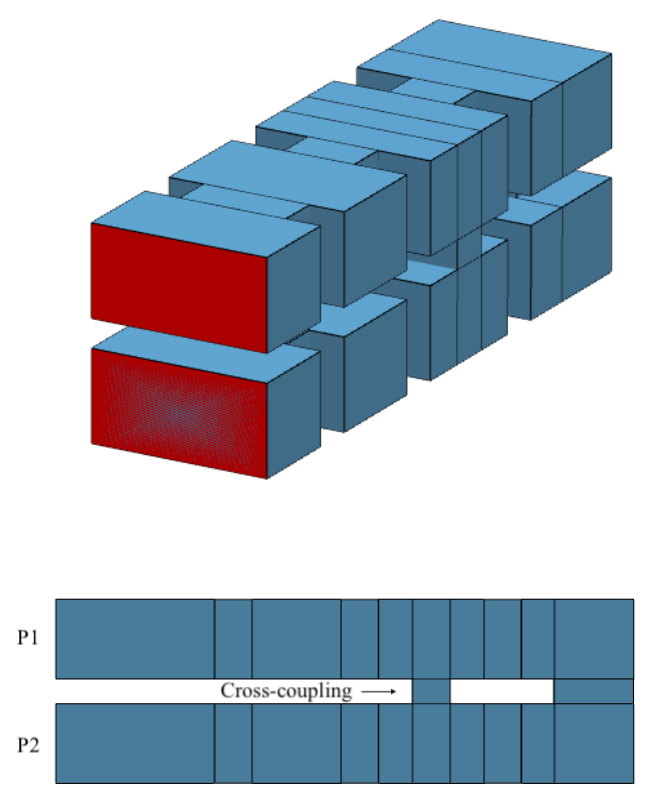

Fig. 3: Top: Perspective view of the folded topology with a cross-coupling window between the second and fifth resonator that will be used for each of the channels. Bottom: Side view of the filter.

2) The filters can be coupled directly to the manifold. There is no need to use additional stubs to enforce length constraints and, therefore, the structure is much more compact.

3) Removing the length constraint increases the degrees of freedom in the design and, in addition, the optimization becomes easier.

4) Cross-coupling windows can be easily implemented between non-adjacent resonators, so that transmission zeros can be implemented in every channel to increase the selectivity.

\section{SpecificAtions AND DESIGN Procedure}

The specifications that we have used as a target for our design are as follows:

- Input/Output waveguide: WR-75 $(a=19.050 \mathrm{~mm}$, $b=9.525 \mathrm{~mm}$ ).

- The channels are centered at 11, 12 and $13 \mathrm{GHz}$.

- The bandwidth are 200, 300 and $400 \mathrm{MHz}$, respectively.

- The order of each channel: $N=6$.

- Every filter has two transmission zeros, one below and one above the pass band.

The filter will be manufactured by milling in three parts: one body and two covers. It is now important to note that, from a theoretical point of view, there are no bandwidth or center frequency restrictions so that any required value can be easily accommodated (with the usual differences between the design of contiguous and non-contiguous multiplexers). 
However, since it is well known that there is a link between the quality factor of the resonators used in a filter, the return loss behavior that can be achieved, and the bandwidth of the filter, we decided to use channels with bandwidth wider than $200 \mathrm{MHz}$ (which corresponds to a relative bandwidth of $3.73 \%$ ). This choice was, in fact, supported by the fact that we manufactured and measured several test filters. The results obtained clearly indicated that filters with a narrower bandwidth could not be tuned to obtain a proper equiripple return loss response.

To continue, we can now summarize the complete design process as follows:

1) Design each of the filter channels independently. A step by step guide on how to design the filters starting from the desired Chebyshev response, and ending with the folded waveguide filters with two transmission zeros is discussed in Section IV.

2) Add the filters to a double manifold and follow an optimization process based on the well-known design procedure described in [12] for manifold multiplexers. Section $\mathrm{V}$ describes in detail the new multi-band filter optimization strategy we have used to design the tri-band filter.

In the design process, we will use two simulators: FEST3D from Aurorasat (now with Dassault Systèmes) and CST Microwave Studio (also with Dassault Systèmes).

FEST3D is a full-wave simulator that uses a multimode equivalent network (MEN) to represent the filter building blocks. One of the basic features of FEST is that the user can choose the set of numerical parameters that define the accuracy, and the computational efficiency. For our initial design, FEST3D will be used in a low-accuracy mode. This setup allows to perform simulations in a very short time. The computational parameters are chosen as follows:

- Number of accessible modes $=10$.

- Number of basis functions $=30$.

- Green's functions terms $=300$.

A detailed explanation of the meaning of each parameter can be found in [14].

Since FEST3D is a powerful simulation tool that can simulate waveguide components in a very short amount of time, in our design process we optimized directly the complete waveguide structure of the multi-band filter. However, if a software tool like FEST3D is not available, the optimization of the multi-band filters can be easily performed using equivalent circuit models of the filters and the manifolds in a circuit simulator such as Keysight Advanced Design System (ADS) to obtain the desired target performance. A final optimization can then be easily carried out to go from the equivalent circuit models to the multi-band filter structure in waveguide technology.

Finally, once we obtained the desired response in the lowaccuracy waveguide model, we use the Aggressive Space Mapping (ASM) procedure to obtain the final high-precision dimensions of the structure [15], [16]. The high accuracy simulations have been performed using CST Microwave Studio.

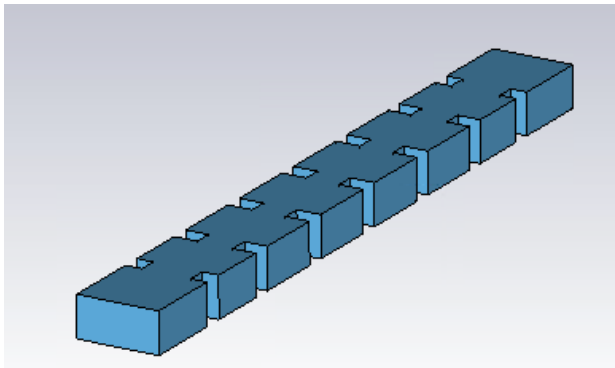

Fig. 4: In-line filter without transmission zeros.

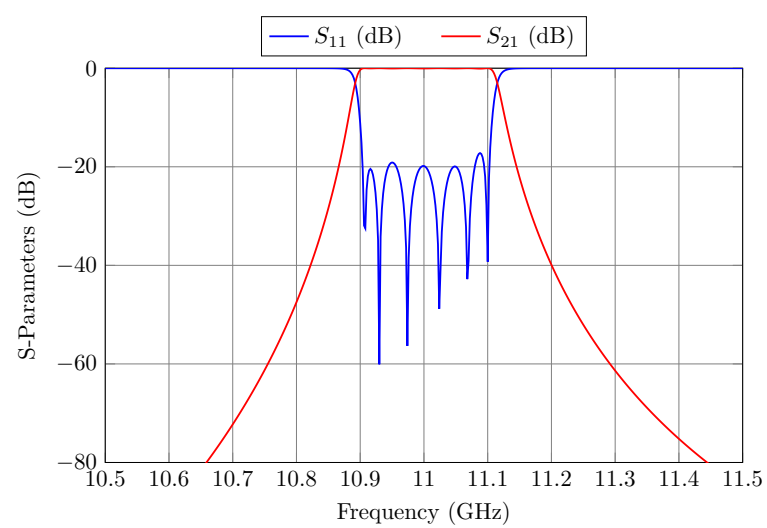

Fig. 5: In-line filter response.

\section{INDEPENDENT CHANNELS}

The design process begins with the independent channel filters (as in [12] and [3]).

\section{A. Folded Filters without TZs}

The first step is to design the in-line filter shown in Fig. 4. To do so, we first design a filter based on transmission lines and inverters that provides the desired Chebyshev response [17]. After that, we follow the synthesis method described in [18], and obtain the waveguide filter of Fig. 4, with the response shown in Fig. 5.

Once the in-line filter has been designed, the next step consists in folding the filter structure in the center. Fig. 6 shows the central coupling window of both the in-line, and the folded filter. After this step, we need to optimize again the dimensions of the folded window (Fig. 6, b) until the same response of the in-line window (Fig. 6, a) is recovered. There are three optimization parameters that can be used for this: the height, the width, and the thickness of the window. The thickness of the coupling window will determine the physical separation between both sides of the filter. It must therefore be chosen to ensure the structural integrity of the filter. We have therefore fixed the height and the thickness of the folded coupling window of the three filter channels to $9.525 \mathrm{~mm}$ and $3 \mathrm{~mm}$, respectively. The width, on the other hand, is optimized to recover the required coupling level.

After finding the optimum dimensions of the folded coupling window, we can add the rest of the filter (see Fig. 7). The exact same performance of Fig. 5 can be easily obtained with a rapid final optimization of the rest of the parameters. 
(a)

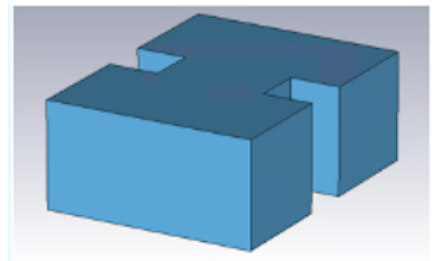

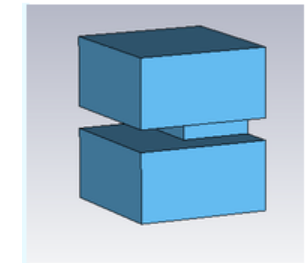

(b)
Fig. 6: (a) Central window of the in-line filer. (b) Central window of the folded filter.

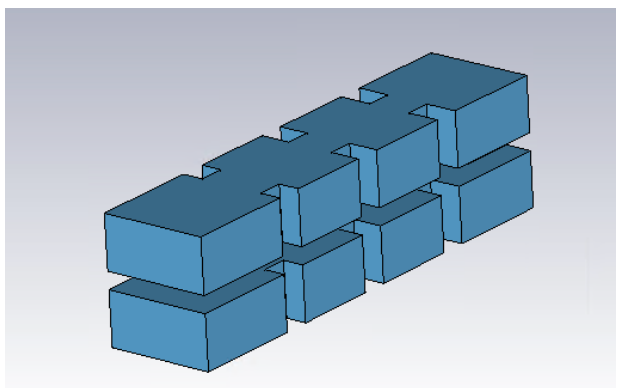

Fig. 7: Folded filter without transmission zeros.

\section{B. Folded Filters with TZs}

The next step in the design of the folded channel filter is to open a cross-coupling window between two non-adjacent resonators. Opening a capacitive coupling window between the second and fifth resonator will introduce one transmission zero below, and one transmission zero above the filter pass band [19]. Fig. 3 shows the channel filter with the capacitive coupling. The thickness of the window is fixed to $3 \mathrm{~mm}$ (the same value of the folded coupling window). The coupling level is controlled by the height.

Although it is possible to obtain the height of this coupling iris starting from the required coupling value, we have chosen to use a simpler, more general and direct procedure, namely:

Step 1: Start with a very small capacitive window, for example, $0.5 \mathrm{~mm}$ high.

Step 2: Optimize only the length of the resonators connected to the cross-coupling window to recover the correct in-band performance.

Step 3: To achieve the desired in-band performance we now need to optimize also the widths of the inductive windows that are close to the cross-coupling window. After this step, we will have recovered the in-band response and we will clearly see the two transmission zeros.

Step 4: At this point, depending on the specific filter structure, we may need to perform a rapid final optimization of all the filter parameters to recover the exact equiripple response.

Step 5: Finally, increase the height of the capacitive window and go back to step 2 .

This procedure is followed until the TZs are located at the desired frequency. In this particular case, the target perfor-

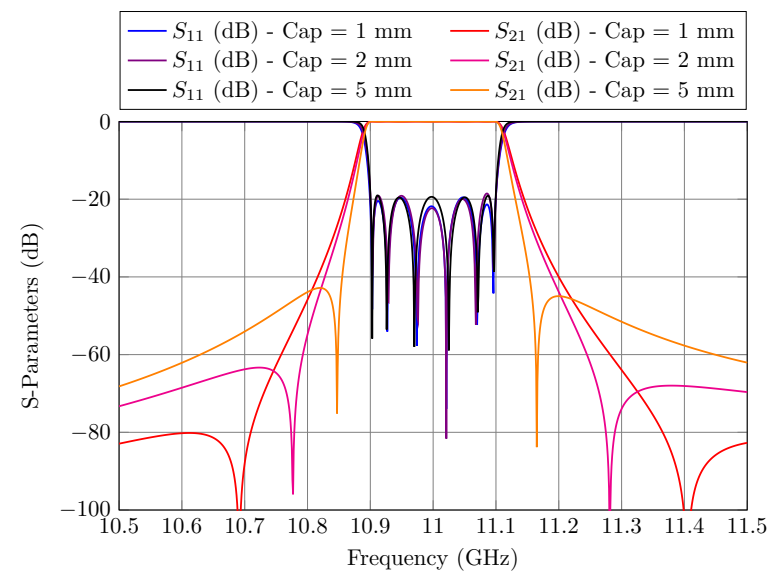

Fig. 8: Filter response for different heights of the coupling window.

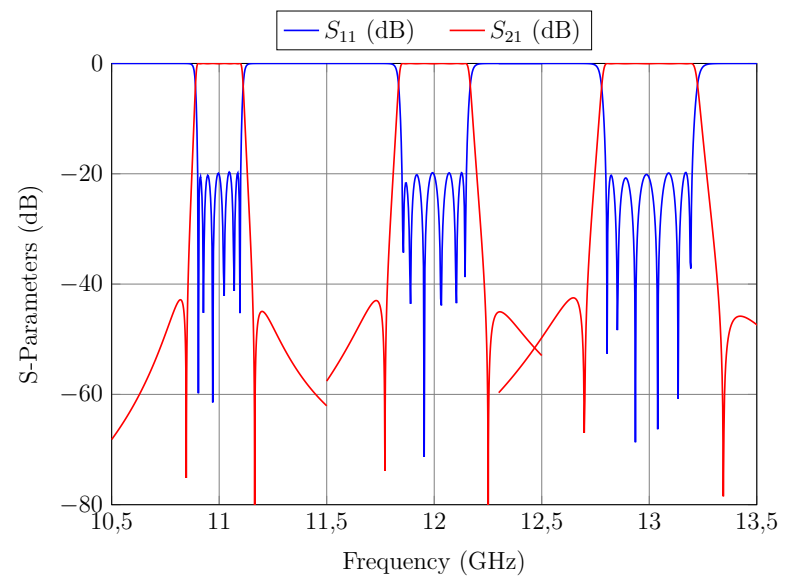

Fig. 9: Filter response for every channel after introducing the TZs.

mance is obtained with a height equal to $4.5 \mathrm{~mm}$. Fig. 8 shows the filter response after step 3 , for three different heights.

This process is repeated for each channel of the multi-band filter. The final response of each independent channel is shown in Fig. 9. Now that the filter channels have been designed, we can proceed with the next stage of the design procedure.

\section{TRI-BAND FILTER}

In order to design the multi-band filter, we could follow the strategy described in [13]: first design an $N$-channel multiplexer, then build the multi-band filter by cutting the structure along the symmetry axis, joining in the middle two identical halves, and perform a final fine optimization to fulfill the required multi-band filter specifications. However, we could also start designing the multi-band filter directly. In this case, the procedure is as follows:

1) Add the filter with the narrowest pass band to a double manifold terminated in short-circuits (see Fig. 10). Then, optimize first the distance between the short-circuits and the first inductive window of the filter. Fig. 11 shows the response of the filter at this point. 


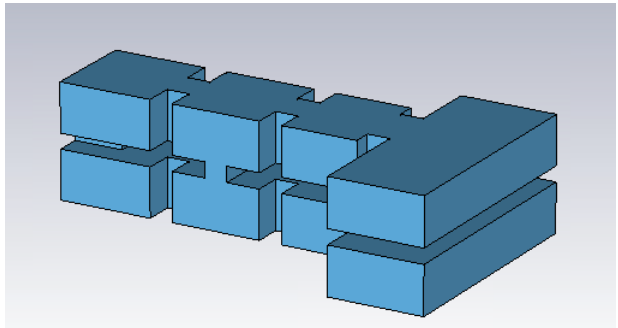

Fig. 10: First filter channel connected to the double manifold.

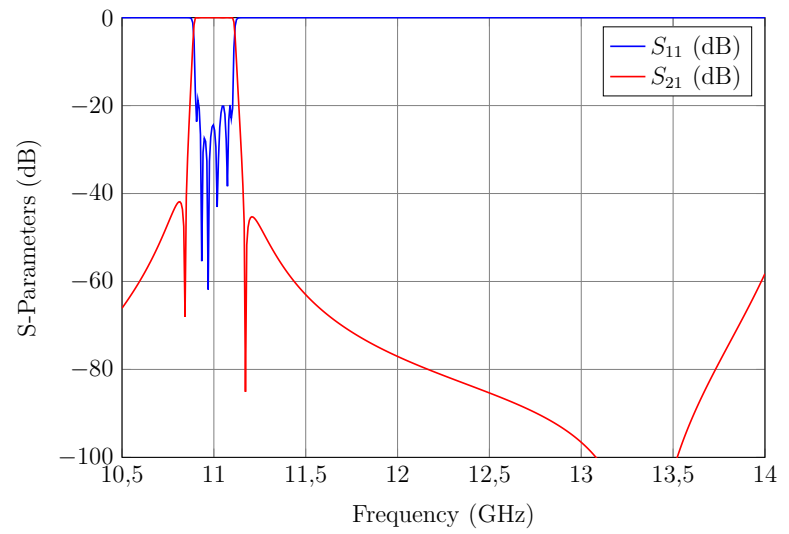

Fig. 11: Performance of the first filter after connecting it to the double manifold.

2) The next step is to add the other channels, one at a time, going from the narrowest bandwidth to the widest bandwidth, proceeding in the same way. Fig. 12 shows the structure of the tri-band filter and Fig. 13 shows its response after adding all the channels.

3) The final step is to follow the classic manifold-based optimization procedure until the desired filter responses are obtained [12]. Fig. 14 shows the optimized response of the multiband filter.

Our investigation indicates that it is indeed important to place the filter with the narrowest bandwidth near the shortcircuited end, and the filter with the widest bandwidth near the input of the manifold. This, in fact, simplifies considerably the optimization process. In physical terms, this can be explained by the fact that, as the filter bandwidth increases, more power is drawn from the manifold into the filter. As a result, placing the filter with the narrowest bandwidth near the short circuit

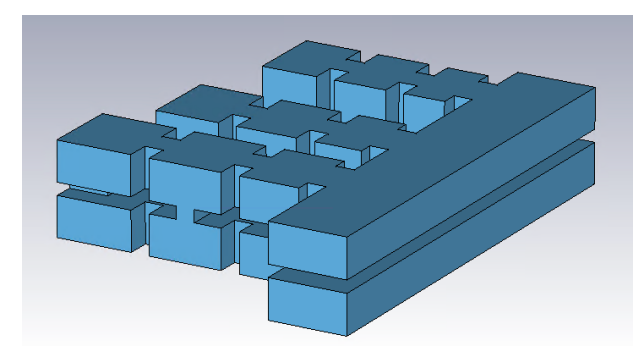

Fig. 12: Structure of the tri-band filter after adding all channels.

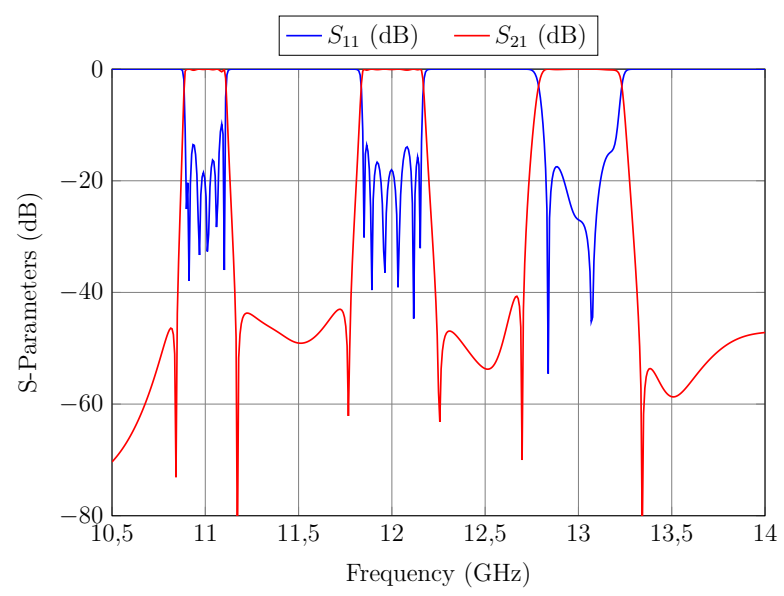

Fig. 13: Performance of the tri-band filter after adding the channels one by one.

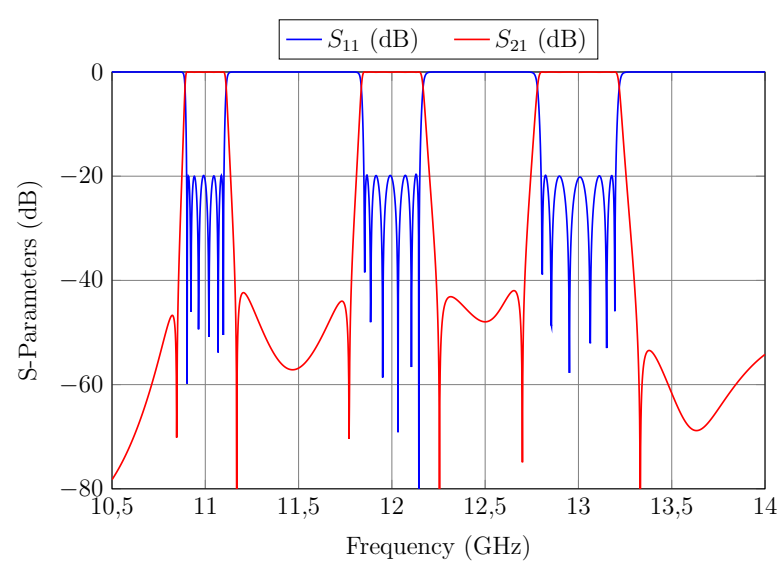

Fig. 14: Performance of the tri-band filter after optimizing its dimensions.

helps considerably in obtaining a first coupling window with a size that can be easily manufactured. Using instead the widest bandwidth filter in the same position, may result in an unsuccessful optimization process either not reaching the specifications or obtaining extreme or non-practical physical dimensions.

Our work indicates that this strategy is more efficient than the one used in [13], because the multi-band filter is built directly, and thus, the final fine optimization to fulfill the required performance is no longer needed.

\section{PRACTICAL CONSIDERATIONS}

The filter is manufactured in three parts: the body and two covers. The body of the filter is manufactured by milling, and M4 screws will be used to assemble the parts. In order to minimize the insertion losses, a pressure well is introduced around each filter to ensure perfect electrical contact between body and cover(s), as shown in Fig. 15.

During the design process, we noticed that the length of the manifold connecting the second and third channel is too short to properly include the closing screws. For that reason, we decided to move the central channel to the other side of 


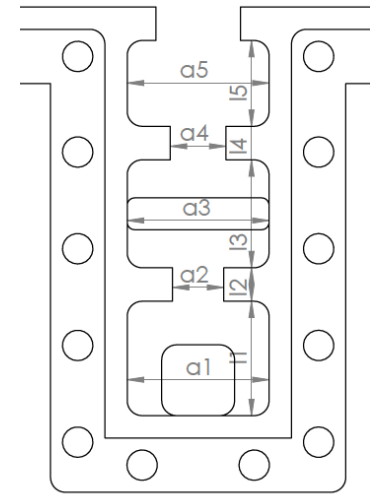

Fig. 15: Pressure well around each channel to perfectly seal the body and covers.

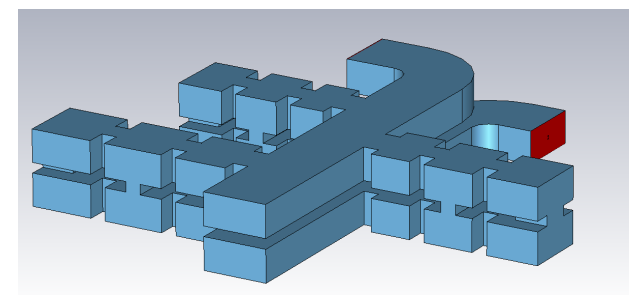

Fig. 16: The central channel has been moved so that the M4 closing screws could be added. H-plane bends have also been added for feeding purposes.

the manifold, as shown in Fig. 16. Note that two H-plane bends have also been added so that we can feed the filter with standard WR-75 flanges. Fig. 17 shows the response of the new filter with the H-plane bends. The electrical response barely changes after moving the central channel and adding the bends. Any slight deviation can be easily compensated with a rapid optimization.

\section{High Precision Simulations}

A yield analysis of the filter has then been performed to determine if this filter requires the use of tuning elements.

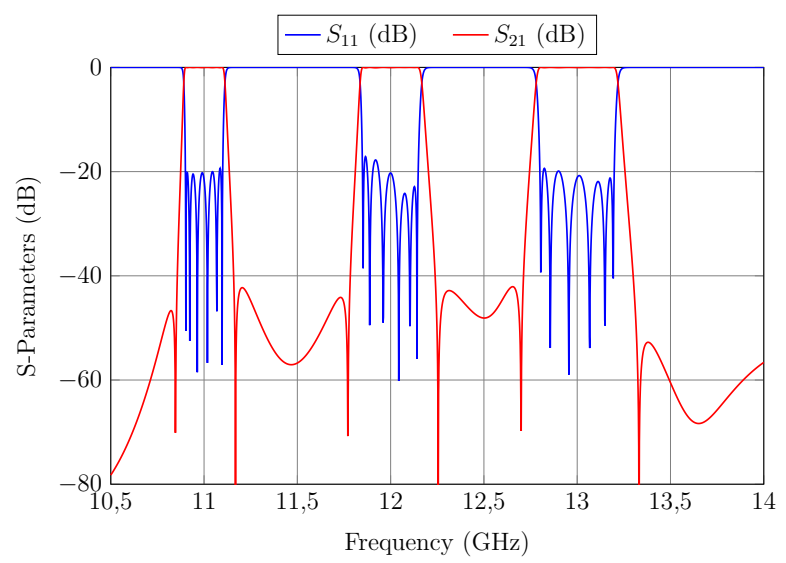

Fig. 17: Filter response after moving the central filter, and adding the H-plane bends.
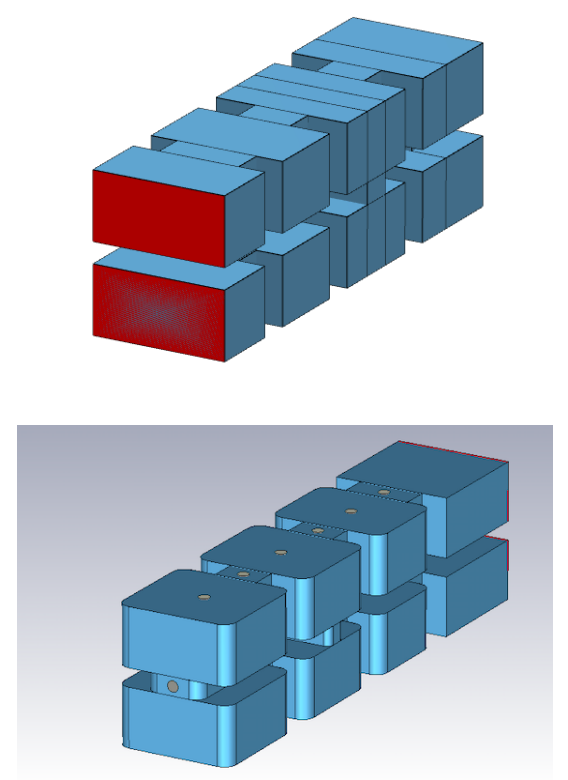

Fig. 18: Top: Extracted channel from the multi-band filter. The simulation of this structure will provide the reference response that the high precision model should match. Bottom: Channel that includes the manufacturing details (round corners and tuning elements).

Using a normal distribution of mean $\mu=0$ and standard deviation of $10 \mu \mathrm{m}$, we obtain a data-set of 300 vectors that represent the manufacturing errors. Only 6 out of the 300 vectors of the data-set comply with the specifications ( $2 \%$ manufacturing yield). This clearly indicates that the filter requires tuning elements. Standard tuners with a radius of $0.9 \mathrm{~mm}$ have, therefore, been included in every cavity, and every inductive window, so that the manufacturing errors can be easily compensated. However, no tuning elements have been included in the capacitive windows.

The filter has then been simulated again including all tuners at a fixed depth of $1 \mathrm{~mm}$. This penetration will ensure a broad margin to tune manually the filter. At this point, we have also included in the simulations the rounded corners in the cavities to account for the milling process. All simulations have been carried out at this stage with CST using a high precision (HP) setup.

The transition between the ideal high precision structure without tuning elements and with sharp corners has been performed using a new approach that combines the ASMbased technique of [16] and the traditional synthesis method described in [18]. The new procedure is as follows:

1) The first step is to extract the individual filters of the ideal, high precision, multi-band filter. The isolated response of each filter will be the reference that the high precision simulation with round corners and tuning elements should reproduce (Fig. 18).

2) For each channel, we next follow the procedure described in [18]. To do so, we first simulate the first three 

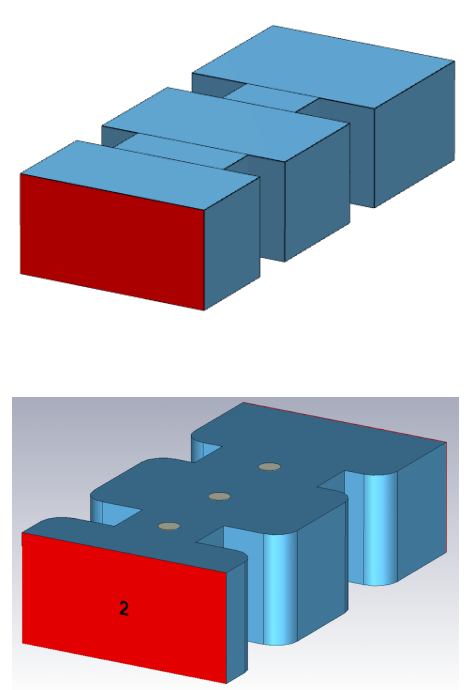

Fig. 19: Top: Reference response of the first three elements. Bottom: High precision model that includes both tuning elements and round corners.

elements of the reference filter: the first window, the first cavity and the second window (Fig. 19, top). Instead of directly optimizing the bottom structure of Fig. 19 to match the desired response of the reference circuit, we next exploit the power of ASM-based technique described in [16]. Since the design parameters of both models are waveguide dimensions, their relation (usually established with a Broyden Matrix in the classical ASM method) can be approximated very well by the identity matrix $(B \approx I)$. Instead of optimizing the slow high precision model (bottom structure of Fig. 19) to recover the reference response (provided by the top circuit of Fig. 19), we will do the opposite: we will optimize the fast low precision (LP) model to recover the initial response of the high precision model. The initial dimensions for the high precision model $\left(x_{\text {fineinit }}\right)$ are the low precision ones $\left(x_{\text {coarseinit }}\right)$.

The optimization will be extremely fast because there are only three parameters. The difference between the initial reference dimensions $\left(x_{\text {coarseinit }}\right)$ and the ones that provide the same performance of the high precision model ( $\left.x_{\text {coarse opt }}\right)$ is:

$$
\Delta=x_{\text {coarseinit }}-x_{\text {coarse opt }}
$$

This difference $\Delta$ is then used to obtain the optimum dimensions of the high precision model:

$$
x_{\text {fineopt }}=x_{\text {fineinit }}+\Delta
$$

After only one iteration of ASM, both models provide the same response (see Fig. 20).

3) Once the same performance is obtained for the first part of the filter, we add the next resonator and the next iris to the high precision model (see Fig. 21). The top structure of Fig. 21 provides again the reference performance for this second stage. We now proceed in the same

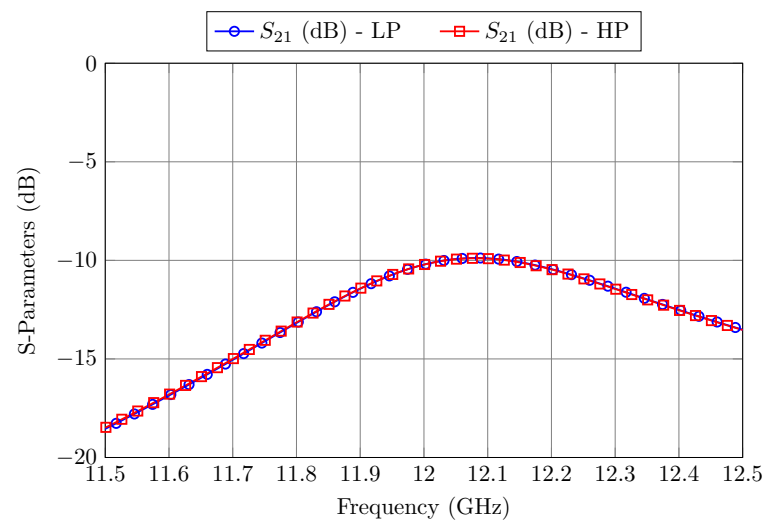

Fig. 20: Transmission response of the circuits represented in Fig. 19. After one ASM iteration, both models provide the same performance.
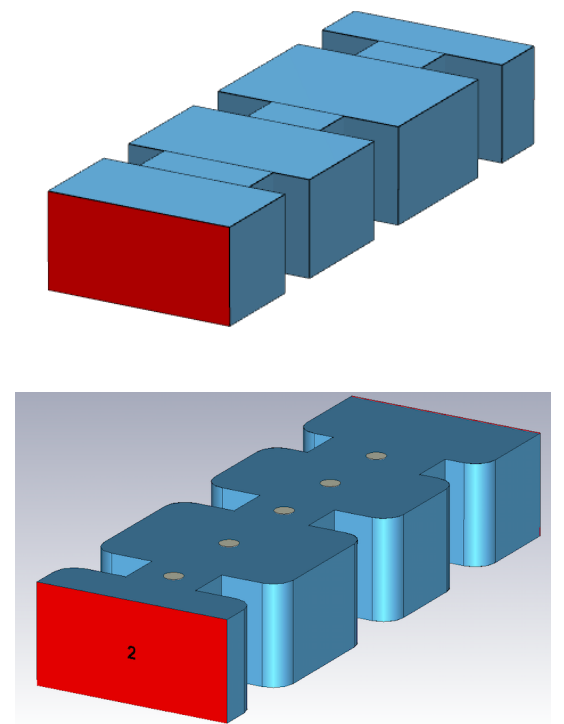

Fig. 21: Top: Reference response of the second stage. Bottom: High precision model that includes both tuning elements and round corners.

way: we optimize only the newly added elements of the fast, low precision model (top of Fig. 21) to recover the performance provided by the high precision model (bottom of Fig. 21). This optimization is even faster than before, since only two parameters are being optimized. After a single iteration of the ASM method, both models provide, essentially, the same desired performance (see Fig. 22).

4) We repeat Step 3 until we reach the center of the filter. Then, since the structure is symmetric, we simply duplicate the structure and we add the capacitive crosscoupling. One final ASM-based optimization is then performed using the models shown in Fig. 18. As in Step 3, we only optimize the newly added elements, that is, the height of the capacitive window and the length of the resonator to which it is connected. Fig. 23 shows the performance of both models after just one 


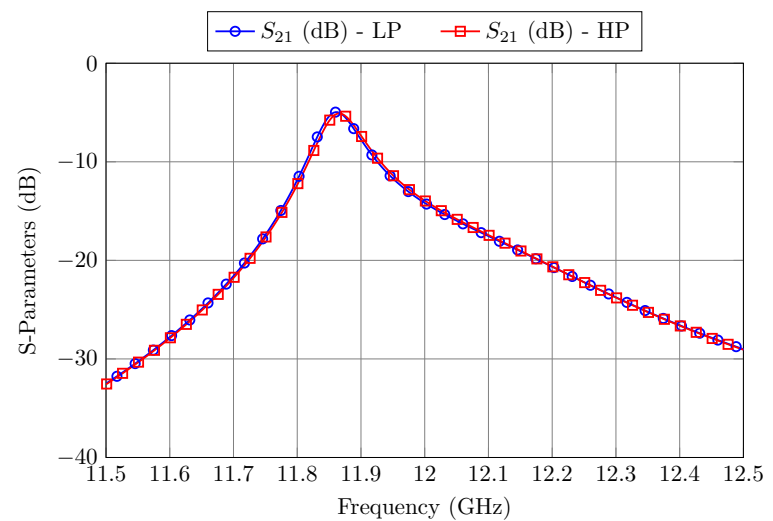

Fig. 22: Transmission response of the circuits represented in Fig. 21. After one iteration of ASM, both models provide the same performance.

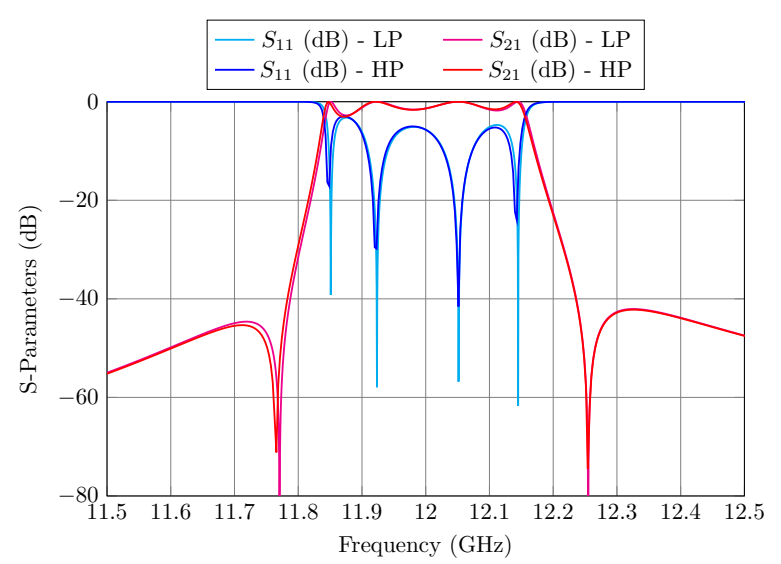

Fig. 23: Performance of the two filters shown in Fig. 18. After one iteration of ASM, both models provide the same performance.

ASM iteration.

5) After doing this for each filter, we can add them together in the manifold obtaining the structure of Fig. 24. The initial response of the filter is shown in Fig. 25.

6) The last step is a final ASM iteration, as described in [16], using the coarse model shown in Fig. 16. Since the initial response of the high precision model is close to the desired one, the optimization will process is very fast even though there are many parameters to

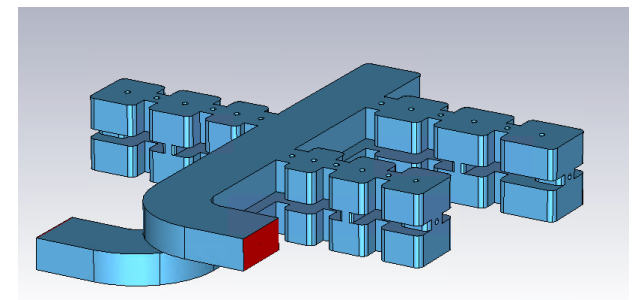

Fig. 24: High precision model of the multi-band filter that considers both round corners and tuning elements.

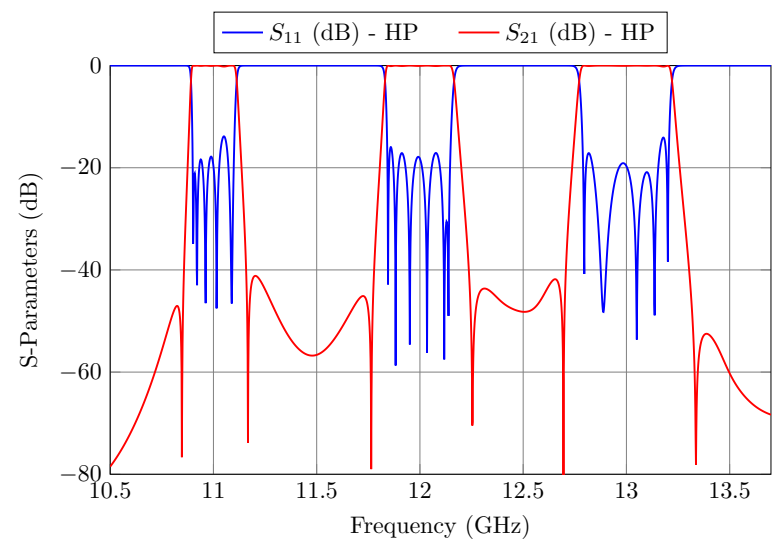

Fig. 25: Initial performance of the high precision multi-band filter shown in Fig. 24.

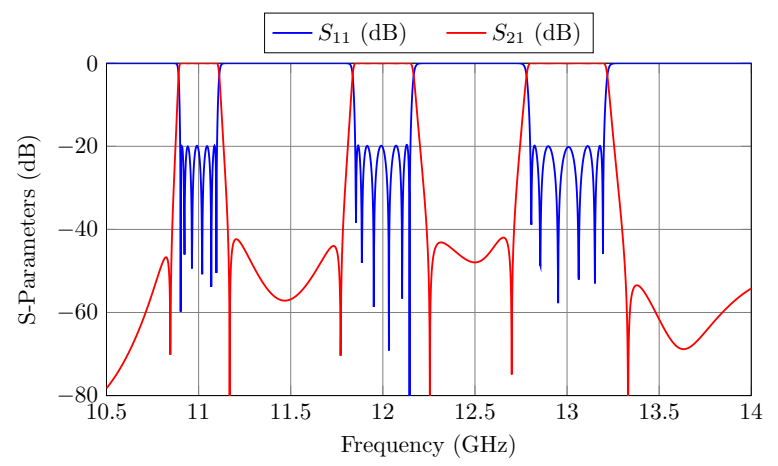

Fig. 26: Final high precision response of the tri-band filter shown in Fig. 24.

optimize. The final performance of the fine model is shown in Fig. 26. The performance of the high precision model that includes round corners and tuning elements is now exactly the same as the one obtained from the low precision model.

If we tried to include the round corners and tuning elements at once in the high precision model (following the ASM-based approach of [16]), the difference between the response of the fine simulator and the goal performance would have been exceedingly large and the coarse model would not be able to reproduce accurately the performance of the fine model. Multiple ASM iterations would then be needed and therefore, the design process would be significantly slower. Table I shows a time comparison between the two approaches. The overall time required to reach the optimal high precision performance with the ASM method described in [16] is computed as:

$$
\text { Total }=T F \cdot(N I+1)+T C \cdot A I \cdot N I
$$

Where $T F$ and $T C$ are, respectively, the computation times of the fine and coarse models, $N I$ is the number of iterations performed and $A I$ is the average number of simulations performed by a Simplex algorithm to recover each of the fine model responses. 
TABLE I: Performance of both design methods.

\begin{tabular}{|c|c|c|c|c|c|c|c|}
\hline \multicolumn{2}{|c|}{ ASM-approach of [16] } & \multicolumn{6}{|c|}{ New proposed method } \\
\hline$T C$ & $0,26 \mathrm{~s}$. & $T C_{1}$ & $0,1 \mathrm{~s}$. & $\overline{T F_{1}}$ & $19 \mathrm{~s}$. & $\overline{I_{1}}$ & 66 \\
\hline$T F$ & $194 \mathrm{~s}$. & $T C_{2}$ & $0,11 \mathrm{~s}$. & $T F_{2}$ & $47 \mathrm{~s}$. & $I_{2}$ & 82 \\
\hline$N I$ & 11 & $T C_{3}$ & $0,12 \mathrm{~s}$. & $T F_{3}$ & $91 \mathrm{~s}$. & $I_{3}$ & 127 \\
\hline$A I$ & 1532 & $T C_{4}$ & $0.26 \mathrm{~s}$. & $T F_{4}$ & $194 \mathrm{~s}$. & $I_{4}$ & 91 \\
\hline & & $T C_{5}$ & $0,26 \mathrm{~s}$. & $T F_{5}$ & $194 \mathrm{~s}$. & $I_{5}$ & 384 \\
\hline Total & 1 h. 52 min. & Total & \multicolumn{5}{|c|}{17 min. $30 \mathrm{~s}$. } \\
\hline
\end{tabular}

The time needed to obtain the desired performance following the newly proposed method is computed as:

$$
\text { Total }=\sum_{i=1}^{\text {Stages }} T C_{i} \cdot I_{i}+2 \cdot T F_{i},
$$

where, $T F_{i}$ and $T C_{i}$, respectively refer to the computation times of the fine and coarse models in every stage, and $I_{i}$ indicates the number of simulations performed by a Simplex algorithm to recover each of the fine model responses. The newly proposed method has proved to be 6.3 times faster than the method reported in [16].

The advantages of this new method are as follows:

- The structure is built step by step, and so, the optimization space in each stage is limited to two or three parameters at the most.

- The method is very robust. Due to the reduced optimization space, the optimizer will not be trapped in a local minimum. The step by step approach, in addition to increasing the robustness of the method, also reduces very considerably the time needed for the complete design process.

- All manufacturing details, such as round corners or tuning elements, can be easily included in the design.

This new strategy is then established as the best option to increase the precision of the simulations and include manufacturing details such as tuners or round corners. However, for structures that do not include these features, the procedure described in [16] may be the simplest way to go.

\section{EXPERIMENTAL VALIDATION}

The tri-band filter was then manufactured in three parts by milling a block of aluminum. A a $10 \mu \mathrm{m}$ silver plating finish was also added to reduce the effect of Ohmic losses. Fig. 27 shows the parts and Fig. 28 shows the assembled filter, already connected to the WR-75 waveguides.

Fig. 29 shows the final measured response of the filter, after the tuning process, compared to the simulated lossless response in the single-mode range of waveguide WR-75. As we can see, there is an excellent agreement between both responses. There are two spurious signals at 14.48 and $14.88 \mathrm{GHz}$. However, their attenuation is greater than $30 \mathrm{~dB}$. The measured insertion losses at the central frequency of the channels $(11,12$ and $13 \mathrm{GHz})$ are $0.49,0.21$ and 0.17 $\mathrm{dB}$, respectively. Fig. 30 shows the measured insertion loss compared to the simulated response in CST.
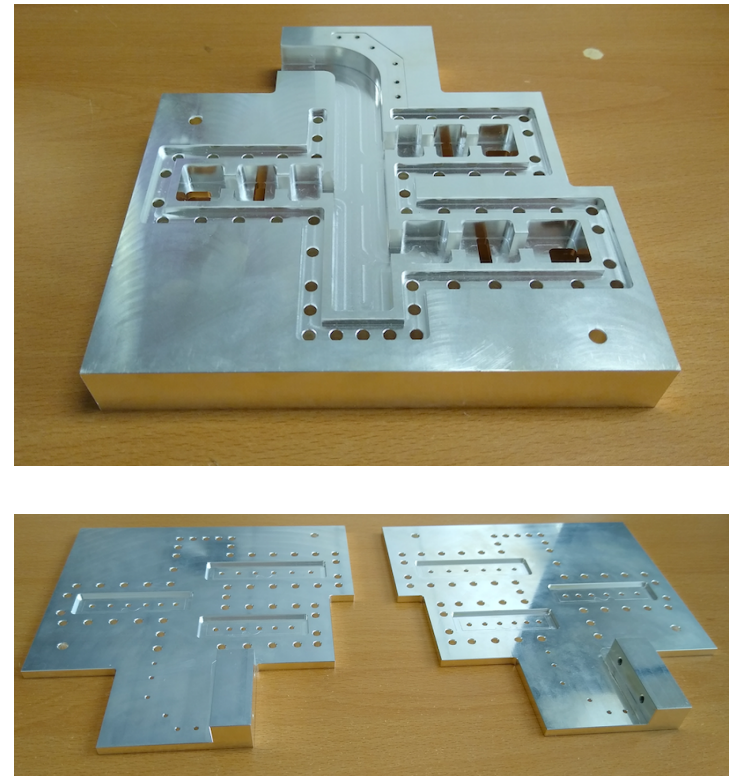

Fig. 27: Top: Body of the tri-band filter. Bottom: Top and bottom covers of the filter.

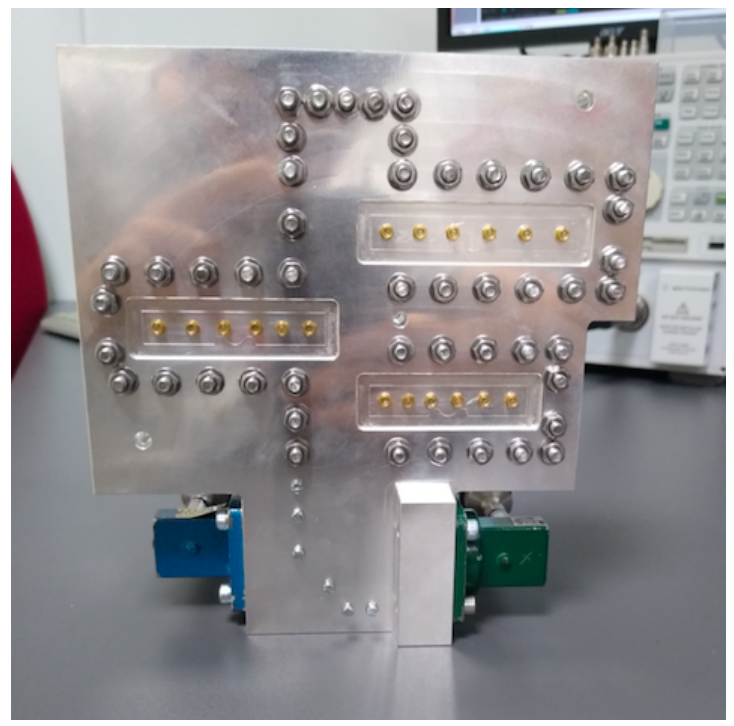

Fig. 28: Assembled tri-band filter already fed with the WR-75 waveguides.

\section{CONCLUSION}

A new topology for multi-channel filters has been proposed based on folded waveguide filters connected to two manifolds. This topology allows to use filters of different length for each channel, and to easily introduce transmission zeros in the response of each separated channel.

In addition, we describe a systematic design procedure based on a well-known technique used to design manifold multiplexers. An efficient ASM-based technique has also been described to go from the low accuracy design to the high precision design.

As a validation, a tri-band filter with different bandwidths has been designed and manufactured. The measured results 


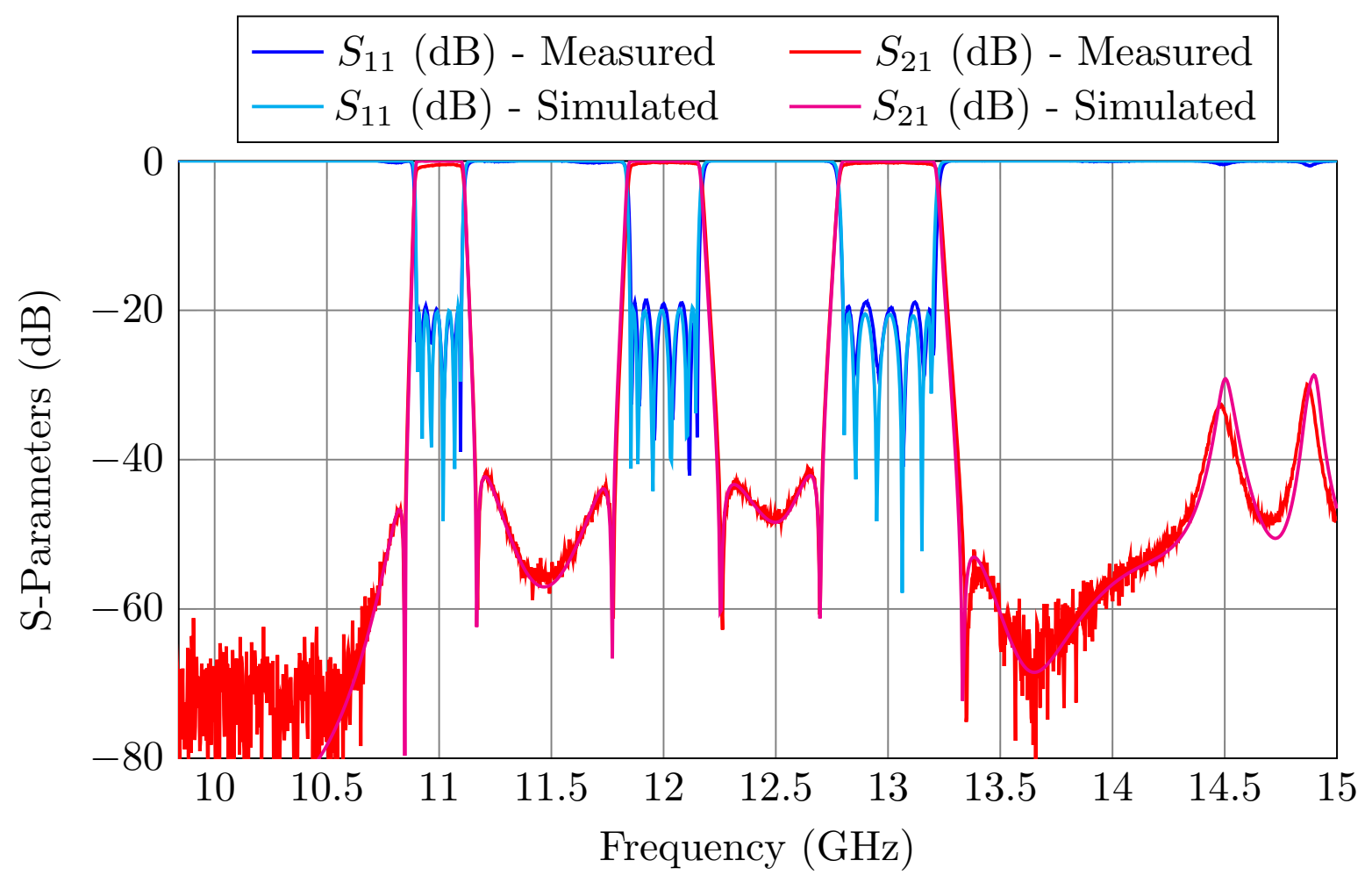

Fig. 29: Measured response of the filter compared to the lossless simulations.

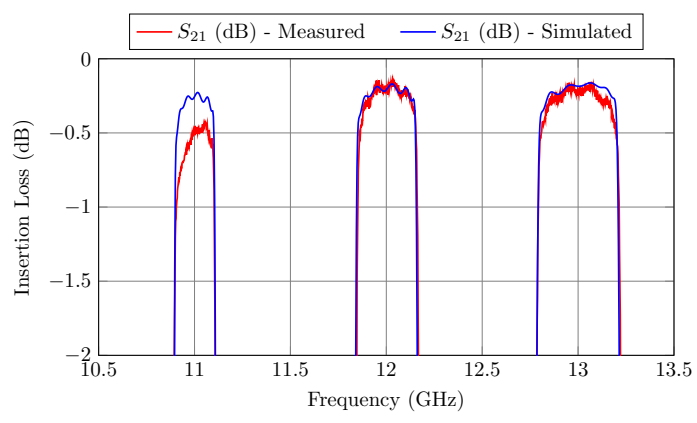

Fig. 30: Measured response of the insertion loss of the filter compared to the simulated losses in CST.

show excellent agreement with the simulations thereby fully validating both the filter topology and the design methodology.

The authors believe that the new proposed folded topology is the best approach to design multi-band filters in waveguide technology in terms of flexibility. This is because the process described has no central frequency or bandwidth restrictions, and transmission zeros can also be easily implemented. Furthermore, it is, in our opinion, the best approach also in terms of scalability. This is because the optimization strategy that we use has been already proved in countless occasions to design multiplexers with a very large number of channels. The same can therefore be easily done to design multi-band filters with many channels.

\section{REFERENCES}

[1] S. Holme, "Multiple passband filters for satellite applications," in 20th AIAA International Communication Satellite Systems Conference and Exhibit, May 2002.

[2] M. Uhm, K. Ahn, I. Yom, and J. Kim, "A triple-passband waveguide filter with dual-mode resonators for Ka band satellite applications," in 24th AIAA International Communications Satellite Systems Conference, June 2006.

[3] R. J. Cameron, C. M. Kudsia, and R. R. Mansour, Microwave Filters for Communication Systems: Fundamentals, Design and Applications., 2nd ed. Hoboken, New Jersey: John Wiley \& Sons, 2018.

[4] L. Zhu, R. R. Mansour, and M. Yu, "Triple-band cavity bandpass filters," IEEE Trans. Microw. Theory Techn., vol. 66, no. 9, pp. 4057-4069, Sep. 2018.

[5] G. Macchiarella and S. Tamiazzo, "Design techniques for dual-passband filters," IEEE Trans. Microw. Theory Techn., vol. 53, no. 11, pp. 32653271, Nov 2005.

[6] M. Mokhtaari, J. Bornemann, K. Rambabu, and S. Amari, "Couplingmatrix design of dual and triple passband filters," IEEE Trans. Microw. Theory Techn., vol. 54, no. 11, pp. 3940-3946, Nov 2006.

[7] J. A. Ruiz-Cruz, M. M. Fahmi, and R. R. Mansour, "Dual-resonance combline resonator for dual-band filters," in IEEE MTT-S Int. Microw. Symp. Dig., June 2012, pp. 1-3.

[8] — "Triple-conductor combline resonators for dual-band filters with enhanced guard-band selectivity," IEEE Trans. Microw. Theory Techn., vol. 60, no. 12, pp. 3969-3979, Dec 2012.

[9] S. Amari and M. Bekheit, "A new class of dual-mode dual-band waveguide filters," IEEE Trans. Microw. Theory Techn., vol. 56, no. 8 , pp. 1938-1944, Aug 2008.

[10] L. Zhu, R. R. Mansour, and M. Yu, "Compact waveguide dual-band filters and diplexers," IEEE Trans. Microw. Theory Techn., vol. 65, no. 5, pp. 1525-1533, May 2017.

[11] _ "A compact waveguide quasi-elliptic dual-band filter," in IEEE MTT-S Int. Microw. Symp. Dig., June 2019, pp. 1179-1182.

[12] R. J. Cameron and M. Yu, "Design of manifold-coupled multiplexers," IEEE Microwave Magazine, vol. 8, no. 5, pp. 46-59, Oct 2007.

[13] S. Cogollos, P. Micó, J. Vague, V. E. Boria, and M. Guglielmi, "New design methodology for multiband waveguide filters based on 
multiplexing techniques," in IEEE MTT-S Int. Microw. Symp. Dig., June 2017, pp. 741-744.

[14] G. Conciauro, M. Guglielmi, and R. Sorrentino, Advanced Modal Analysis. Chichester: John Wiley \& Sons, 2000.

[15] J. W. Bandler, R. M. Biernacki, S. H. Chen, R. H. Hemmers, and K. Madsen, "Electromagnetic optimization exploiting aggressive space mapping," IEEE Trans. Microw. Theory Techn., vol. 43, no. 12, pp. 2874-2882, Dec. 1995.

[16] J. Ossorio, J. C. Melgarejo, V. E. Boria, M. Guglielmi, and J. W. Bandler, "On the alignment of low-fidelity and high-fidelity simulation spaces for the design of microwave waveguide filters," IEEE Trans. Microw. Theory Techn., vol. 66, no. 12, pp. 5183-5196, Dec. 2018.

[17] D. M. Pozar, Microwave Engineering, 4th ed. Hoboken, New Jersey: John Wiley \& Sons, 2011.

[18] M. Guglielmi and A. A. Melcon, "Novel design procedure for microwave filters," in 1993 23rd European Microwave Conference, Sep. 1993, pp. $212-213$.

[19] J. B. Thomas, "Cross-coupling in coaxial cavity filters - a tutorial overview," IEEE Trans. Microw. Theory Techn., vol. 51, no. 4, pp. 1368 1376, April 2003

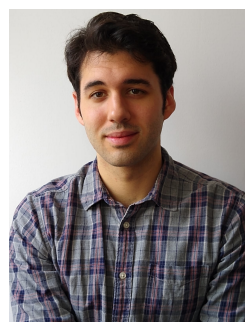

Juan Carlos Melgarejo was born in Alicante, Spain in 1993. He obtained his bachelor's degree in Telecommunications from the Universitat Politècnica de València (UPV) in 2015. He continued his studies with the UPV where he obtained a double Master in Telecommunications Systems in 2019. Currently, he is pursuing his Ph.D. at the university where his main research interests have been investigating microwave passive devices and new manufacturing techniques for satellite components.

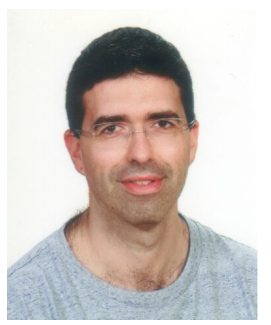

Santiago Cogollos (M'07) was born in Valencia, Spain, on January 15, 1972. He received his "Ingeniero de Telecomunicación" degree and the $\mathrm{Ph}$. D. degree from the Universitat Politècnica de València (UPV), Valencia, Spain, in 1996 and 2002, respectively. In 2000 he joined the Communications Department of the Universitat Politècnica de València, where he was an Assistant Lecturer from 2000 to 2001, a Lecturer from 2001 to 2002, and became an Associate Professor in 2002. He has collaborated with the European Space Research and Technology Centre of the European Space Agency in the development of modal analysis tools for payload systems in satellites. In 2005 he held a post doctoral research position working in the area of new synthesis techniques in filter design at University of Waterloo, Waterloo, Ont., Canada. His current research interests include applied electromagnetics, mathematical methods for electromagnetic theory, analytical and numerical methods for the analysis of microwave structures, and design of waveguide components for space applications.

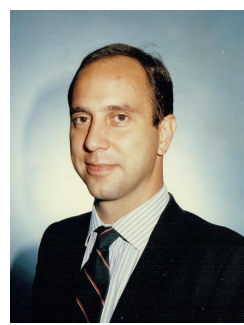

Marco Guglielmi was born in Rome, Italy, on December 17, 1954. He received the degree "Laurea in Ingegneria Elettronica" in 1979 from the University of Rome "La Sapienza", Rome, Italy, where in 1980 he also attended the "Scuola di Specializzazione in Elettromagnetismo Applicato". In 1981 he was awarded a Fulbright Scholarship in Rome, Italy, and an HISP (Halsey International Scholarship Programme) from the University of Bridgeport, Bridgeport, Connecticut, USA, where in 1982 he obtained an MS Degree in Electrical Engineering. In 1986 he received a $\mathrm{PhD}$ degree in Electrophysics from the Polytechnic University, Brooklyn, New York, USA. From 1984 to 1986 he was Academic Associate at Polytechnic University, and from 1986 to 1988 he was Assistant Professor in the same institution. From 1988 to 1989 he was Assistant Professor at the New Jersey Institute of Technology, Newark, New Jersey, USA. In 1989 he joined the European Space Agency as a Senior Microwave Engineer in the RF System Division of the European Space Research and Technology Centre (ESTEC), Noordwijk, The Netherlands, where he was in charge of the development of microwave filters and electromagnetic simulation tools. In 2001 he was appointed Head of the Technology Strategy Section of ESTEC where he contributed to the development of management processes and tools for the formulation of a European strategy for Space Technology Research and Development. In 2014 Dr. Guglielmi retired from the European Space Agency and is currently holding the position of Invited Senior Researcher at the Polytechnic University of Valencia, Valencia, Spain. Dr. Guglielmi has been elevated to the grade of Fellow of the IEEE in January 2013 "For contributions to multimode equivalent networks and microwave filter design".

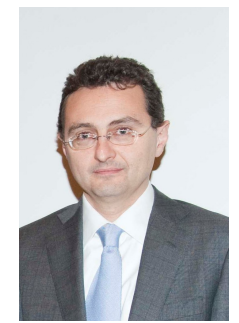

Vicente E. Boria (S'91-A'99-SM'02-F'18) was born in Valencia, Spain, on May 18, 1970. He received his "Ingeniero de Telecomunicación" degree (with first-class honors) and the "Doctor Ingeniero de Telecomunicación" degree from the Universidad Politécnica de Valencia, Valencia, Spain, in 1993 and 1997, respectively. In 1993 he joined the "Departamento de Comunicaciones", Universidad Politécnica de Valencia, where he has been Full Professor since 2003. In 1995 and 1996, he was holding a Spanish Trainee position with the European Space Research and Technology Centre, European Space Agency (ESTEC-ESA), Noordwijk, The Netherlands, where he was involved in the area of EM analysis and design of passive waveguide devices. He has authored or co-authored 10 chapters in technical textbooks, 180 papers in refereed international technical journals, and over 200 papers in international conference proceedings. His current research interests are focused on the analysis and automated design of passive components, left-handed and periodic structures, as well as on the simulation and measurement of power effects in passive waveguide systems. Dr. Boria has been a member of the IEEE Microwave Theory and Techniques Society (IEEE MTT-S) and the IEEE Antennas and Propagation Society (IEEE AP-S) since 1992. He is also member of the European Microwave Association (EuMA), and has been the Chair of the 48th European Microwave Conference held in Madrid, Spain. He acts as a regular reviewer of the most relevant IEEE and IET technical journals on his areas of interest. He has been Associate Editor of IEEE Microwave and Wireless Components Letters (2013-2018) and IET Electronics Letters (2015-2018). Presently, he serves as Subject Editor (Microwaves) of IET Electronics Letters, and as Editorial Board member of International Journal of RF and Microwave Computer-Aided Engineering. He is also member of the Technical Committees of the IEEE-MTT International Microwave Symposium and of the European Microwave Conference. 